\title{
Random walks on the Apollonian network with a single trap
}

\author{
Zhongzhi Zhang ${ }^{1,2}$ (a), Jihong Guan ${ }^{3}$ (b) ${ }^{\text {(a) Wenlei Xie }}{ }^{1,2}$, Yi QI ${ }^{1,2}$ and Shuigeng Zhou ${ }^{1,2}$ (c) \\ 1 School of Computer Science, Fudan University, Shanghai 200433, China \\ ' ${ }^{2}$ Shanghai Key Lab of Intelligent Information Processing, Fudan University, Shanghai 200433, China \\ 3 Department of Computer Science and Technology, Tongji University, 4800 Cao'an Road, Shanghai 201804, China
}

PACS 05.40.Fb - Random walks and Levy flights

PACS 89.75.Hc - Networks and genealogical trees

PACS 05.60.Cd - Classical transport

\begin{abstract}
Explicit determination of the mean first-passage time (MFPT) for trapping problem on complex media is a theoretical challenge. In this paper, we study random walks on the Apollonian network with a trap fixed at a given hub node (i.e. node with the highest degree), which are simultaneously scale-free and small-world. We obtain the precise analytic expression for the MFPT that is confirmed by direct numerical calculations. In the large system size limit, the MFPT approximately grows as a power-law function of the number of nodes, with the exponent much less than 1, which is significantly different from the scaling for some regular networks or fractals, such as regular lattices, Sierpinski fractals, T-graph, and complete graphs. The Apollonian network is the most efficient configuration for transport by diffusion among all previously studied structure.
\end{abstract}

Introduction. - Trapping is an integral major theme of random walks (diffusion) [1-3], which is relevant to a wide range of applications and has led to a growing num' ber of theoretical and practical investigation over the past decades [4-9]. The trapping problem, first introduced ' in [10], is in fact a random-walk issue, where a trap is positioned at a given location, which absorbs all particles visiting it. The primarily interesting quantity closely related to trapping problem is the average trapping time, also referred to as the mean first-passage time (MFPT), which is useful in the study of transport-limited reactions [11,12], target search $[13,14]$ and other physical problems.

An important question in the study of trapping is how the MFPT scales with the size of the system. There are some well-known results providing answers to the corresponding questions in the cases of some graphs with simple topology, including regular lattices [10], Sierpinski fractals [15, 16], T-fractal [17], and so on. However, these graphs are not suitable to describe real systems [18] encountered in everyday experience, most of which are scalefree [19] and small-world [20] that have been shown to influence profoundly various dynamical processes running on networks $[21,22]$. Thus, it is natural and interesting

\footnotetext{
(a) zhangzz@fudan.edu.cn

(b) jhguan@tongji.edu.cn

(c) sgzhou@fudan . edu . cn
}

to explore the trapping problem on networks with general structure embedded in real life. Although a lot of activities have been devoted to studying random walks on complex networks [23-27], work about trapping problem on scale-free small-world graphs is much less [28].

In the paper, we investigate the trapping problem on the Apolloian network $[29,30]$ with scale-free and smallworld properties. We focus on a specific aspect of random walks in the presence of a single trap situated at a given node with the largest degree (hub node). We obtain an exact analytical solution for the MFPT and the dependence of this primary quantity on the system size. We show that the Apolloian network is a preferred architecture that minimizes the increase of MFPT with network size, compared with regular networks and fractals.

Introduction to the Apollonian network. - We first introduce the Apollonian packing [31], from which the Apollonian network is derived. There are two commonly used Apollonian packings that differ mainly in initial configurations. The first packing is constructed by starting with three mutually touching disks, the interstice of which is a curvilinear triangle. In the first generation a disk is inscribed, touching all the sides of this curvilinear triangle. For subsequent generations we indefinitely repeat the packing process for all the new curvilinear triangles. In 

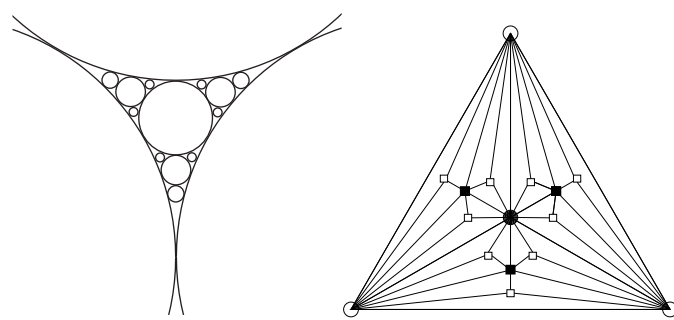

Fig. 1: (Left panel) The first three generations of the first Apollonian packing of disks. (Right panel) An Apollonian network corresponding to the packing shown in the left panel.

the limit of infinite generations, an Apollonian packing is obtained. The left panel of figure 1 displays the first three generations of this Apollonian packing.

The other frequently used Apollonian packing has a initial configuration with three mutually touching disks inscribed inside a circular space. The interstices of the initial disks and circle are four curvilinear triangles to be filled. Then in each subsequent generation, we add one disk to each interstice (curvilinear triangle), so that the added disk touches all the three sides of the corresponding curvilinear triangle. The first several processes are shown in figure 2

The above two Apollonian packings can be easily mapped to networks, usually called as Apollonian networks $[29,30]$. The translation from Apollonian packing construction to Apollonian network generation is quite straightforward. Each node (vertex, site) corresponds to a disk, and two nodes are linked to each other if their corresponding disks are tangent. Note that for the second Apollonian packing, the initial circle also corresponds to a node. The right panel of figure 1 shows an example of the network. Since the resulting networks associated with the two packings have similar structural features, except for the initial disks, in this paper we will focus on the network based on the second Apollonian packing, which is convenient for analytically deriving the network properties.

According to the foregoing mapping, one can introduce a general algorithm $[32,33]$ to create the Apollonian network, denoted by $\mathbb{A}_{g}$ after $g$ generation evolutions. For $g=0, \mathbb{A}_{0}$ is a tetrahedron with four faces or triangles, see figure 3. For $g \geq 1, \mathbb{A}_{g}$ is obtained from $\mathbb{A}_{g-1}$. For each of the existing triangles of $\mathbb{A}_{g-1}$ that is created at generation $t-1$, a new node is added and connected to all the three nodes of this triangle. Figure 4 illustrates the construction process for the first two generations of the initial four faces as shown in figure 3 .

According to the network construction, one can see [30, 33 ] that at the $g_{i}$ th $\left(g_{i} \geq 1\right)$ generation, the number of newly introduced nodes is $L_{v}\left(g_{i}\right)=4 \times 3^{g_{i}-1}$. From this result, we can easily compute the total number of nodes $N_{g}$ in network $\mathbb{A}_{g}$ (hereafter called the network order):

$$
N_{g}=\sum_{g_{i}=0}^{g} L_{v}\left(g_{i}\right)=2 \times 3^{g}+2 .
$$

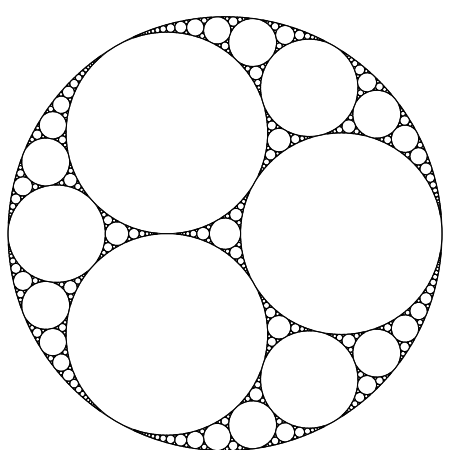

Fig. 2: The first several generations of the second Apollonian packing of disks within a circle.

For convenient use in the next section, we distinguish different nodes of $\mathbb{A}_{g}$ by labeling them sequentially as follows. The four nodes (i.e., nodes $B, C, D$, and $E$ in figure 3) created at initial generation are labeled as $1,2,3$, and 4 , respectively. Then, in each new generation, we only label the new nodes added at this generation, while keep the labels of pre-existing nodes unchanged. That is to say, we label new nodes progressively as $M+1, M+2, \ldots, M+\Delta M$, in which $M$ is the total number of the pre-existing nodes and $\Delta M$ is the number of newly-added nodes. Eventually, every node is labeled by a unique integer, all nodes in $\mathbb{A}_{g}$ are labeled from 1 to $N_{g}$, see figure 4

Let $k_{i}(g)$ be the degree of a node $i$ at generation $g$, which entered the network at the generation of $g_{i}\left(g_{i} \geq 0\right)$. Then $[30,33]$

$$
k_{i}(g)=3 \times 2^{g-g_{i}} .
$$

From equation (2), one can easily see that at each step the degree of a node doubles, i.e.,

$$
k_{i}(g)=2 k_{i}(g-1) .
$$

The Apollonian network presents the typical characteristics of real-life networks $[29,30,32-34]$. It has a powerlaw degree distribution $P(k) \sim k^{-\gamma}$ with the exponent $\gamma=1+\frac{\ln 3}{\ln 2}$. Its average path length, defined as the mean of shortest distance between all pairs of nodes, increases logarithmically with network order [34]. In the large network order limit, the average clustering coefficient tends to 0.8284. Thus, the Apollonian network exhibits smallworld effect [20]. In addition, the network is disassortative [30]. In view of its structural properties similar to those of real networks and its intrinsic interest, Apollonian network is a good substrate network for studying criticality phenomena and dynamical processes [35-43]. In what follows we will investigate the MFPT for random walks with an immobile trap on the Apollonian network.

Formulation of the problem. - In this section we formulate the problem of a simple random walk of a particle on Apollonian network $\mathbb{A}_{g}$ in the presence of a trap or a perfect absorber positioned on a given node. 


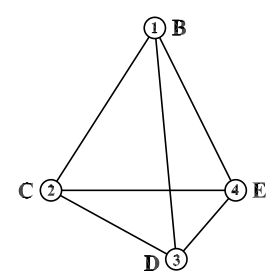

Fig. 3: The initial construction of Apollonian network.

To this end, we first specify $\mathbb{A}_{g}$ by its adjacency matrix $\mathbf{A}_{g}$ (hereafter we neglect the subscript) of order $N_{g} \times N_{g}$, which completely describes network $\mathbb{A}_{g}$. The element $a_{i j}$ of $\mathbf{A}$ is defined as follows: $a_{i j}=1$ if the pair of node $i$ and node $j$ is connected by a link (edge, bond), otherwise $a_{i j}=0$. The degree $k_{i}(g)$ of node $i$ is $k_{i}(g)=\sum_{j=1}^{N_{g}} a_{i j}$, and the diagonal degree matrix $\mathbf{Z}$ is given by $\mathbf{Z}=\operatorname{diag}\left(k_{1}(g), k_{2}(g), \cdots, k_{i}(g), \cdots, k_{N_{g}}(g)\right)$. Then, the normalized Laplacian matrix of $\mathbb{A}_{g}$ is given by $\mathbf{L}=\mathbf{I}-\mathbf{Z}^{-1} \mathbf{A}$ and its entry is $l_{i j}=1-\frac{a_{i j}}{k_{i}(g)}$, where $\mathbf{I}$ is an identity matrix.

Before proceeding further, let us introduce the so-called simple random walk [1-3] on network $\mathbb{A}_{g}$. At each time step (taken to be unity), the walker moves from its current location to any of its nearest neighbors with equal probability. According to this rule, at time $t$, a walker at a node $i$ will hop to one of its $k_{i}(g)$ neighbors, say $u$, with the transition probability $a_{i u} / k_{i}(g)$. Suppose that the walker starts from node $i$ at $t=0$, then the jumping probability $P_{i j}$ of going from $i$ to $j$ at time $t$ is governed by the following master equation:

$$
P_{i j}(t+1)=\sum_{w=1}^{N_{g}} \frac{a_{w j}}{k_{w}(g)} P_{i w}(t) .
$$

We next focus a random walk on $\mathbb{A}_{g}$ with a trap. We locate the trap at node 1 , represented as $i_{T}$. It should be pointed out that thanks to the symmetry, the trap can be also situated at node 2,3 , or 4 , which has no influence on MFPT. The particular selection for the trap location allows one to calculate analytically the MFPT, which will be addressed in detail in the next section. At each time step, the walker, starting from any node except the trap $i_{T}$, jumps to any of its nearest neighbors with equal probability.

Let $T_{i}$ be mean transmit time (first-passage time, trapping time, or the mean time to absorption) for a walker, starting from node $i$, to first arrive at the $\operatorname{trap} i_{T}$. Thus, $T_{i_{T}}=0$. Then, the set of these interesting quantities follows the recurrence equation [44]

$$
T_{i}=\sum_{j=2}^{N_{g}} \frac{a_{i j}}{k_{i}(g)} T_{j}+1,
$$

where $i \neq i_{T}$. Equation (5) may be also rewritten in matrix notation as

$$
\mathbf{T}=\boldsymbol{\Delta}^{-1} \mathbf{e}
$$

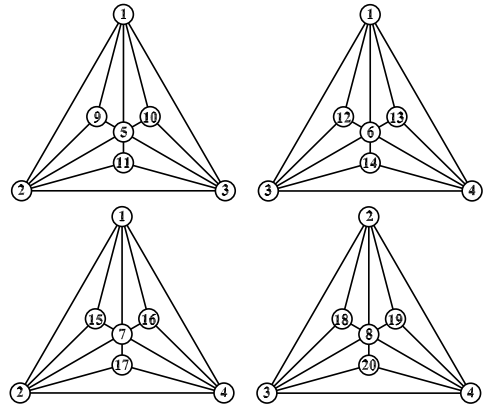

Fig. 4: The Apollonian network of generation 2 and the labeling of its nodes.

where $\mathbf{T}=\left(T_{2}, T_{3}, \cdots, T_{N}\right)^{\top}$ and $\mathbf{e}=(1,1, \cdots, 1)^{\top}$ are two $\left(N_{g}-1\right)$-dimensional vectors, and $\boldsymbol{\Delta}^{-1}$ is the fundamental matrix of the Markov chain representing such unbiased random walk. In fact, $\boldsymbol{\Delta}$ is a sub-matrix of the normalized discrete Laplacian matrix $\mathbf{L}$ whose first row and column, corresponding to the trap node, have been removed.

Then, the mean first-passage time, or the average of the mean time to absorption, $\langle T\rangle_{g}$, which is the average of $T_{i}$ over all nodes distributed uniformly over nodes in $\mathbb{A}_{g}$ other than the trap, is given by

$$
\langle T\rangle_{g}=\frac{1}{N_{g}-1} \sum_{i=2}^{N_{g}} T_{i}=\frac{1}{N_{g}-1} \sum_{i=2}^{N_{g}} \sum_{j=2}^{N_{g}}\left(\Delta^{-1}\right)_{i j} .
$$

Equation (7) can be easily explained by looking at the random walk from the perspective of a Markov chain. Actually, the entry $\left(\Delta^{-1}\right)_{i j}$ of the fundamental matrix $\boldsymbol{\Delta}^{-1}$ of the Markov process expresses the average number of times that a walker starting at node $i$ will be at node $j$, and the row sum $\sum_{j=2}^{N_{g}}\left(\Delta^{-1}\right)_{i j}$ is exactly $T_{i}$, the total times a particle starting at node $i$ will traverse all other nodes before being absorbed by the trap.

Equation (7) shows that the problem of determining $\langle T\rangle_{g}$ is reduced to finding the sum of all elements of the fundamental matrix $\boldsymbol{\Delta}^{-1}$ of order $\left(N_{g}-1\right) \times\left(N_{g}-1\right)$, which can be obtained by utilizing a standard software package, Mathematica 5.0. Irrespective of the seemingly compact expression of equation (7), since $N_{g}$ increases exponentially with $g$, for large $g$, it becomes difficult to obtain $\langle T\rangle_{g}$ through direct calculation from this equation, because of the limitations of time and computer memory. Therefore, one can compute directly the MFPT only for the first generations, see table 1] However, the particular construction of the Apollonian network allows one to calculate analytically MFPT to obtain a rigorous solution. Details of derivation will be provided below.

Exact solution for mean first-passage time. -

Before deriving the general formula for MFPT, $\langle T\rangle_{g}$, we first establish the scaling relation dominating the evolution of $T_{i}^{g}$ with generation $g$, where $T_{i}^{g}$ is the trapping time for a walk originating at node $i$ on the $g$ th generation of Apollonian network. 
Table 2: Mean time to absorption $T_{i}^{g}$ for a random walker starting from node $i$ on the Apollonian network for various $g$. Notice that owing to the obvious symmetry, nodes in a parenthesis are equivalent, since they have the same trapping time. All the values are calculated straightforwardly from equation (7).

\begin{tabular}{l|cccccc}
\hline \hline$g \backslash i$ & $(2,3,4)$ & $(5,6,7)$ & $(8)$ & $(9,10,12,13,15,16)$ & $(11,14,17)$ & $(18,19,20)$ \\
\hline 0 & 3 & & & & & \\
1 & $27 / 5$ & $23 / 5$ & $32 / 5$ & & & $256 / 25$ \\
2 & $243 / 25$ & $207 / 25$ & $288 / 25$ & 7 & $283 / 25$ \\
3 & $2187 / 125$ & $1863 / 125$ & $2592 / 125$ & $63 / 5$ & $2304 / 125$ & $2547 / 125$ \\
4 & $19683 / 625$ & $16767 / 625$ & $23328 / 625$ & $567 / 25$ & $20736 / 625$ & $22923 / 625$ \\
5 & $177147 / 3125$ & $150903 / 3125$ & $209952 / 3125$ & $5103 / 125$ & $186624 / 3125$ & $206307 / 3125$ \\
6 & $1594323 / 15625$ & $1358127 / 15625$ & $1889568 / 15625$ & $45927 / 625$ & $1679616 / 15625$ & $1856763 / 15625$ \\
\hline \hline
\end{tabular}

Table 1: The MFPT obtained by direct calculation from equation (7) by using a standard software package, Mathematica 5.0. Since for large networks, the computation of the MFPT from equation (7) is prohibitively time and memory consuming, we calculate the MFPT for the first several generations.

\begin{tabular}{ccc}
\hline \hline$g$ & $N_{g}$ & $\langle T\rangle_{g}$ \\
\hline 0 & 4 & $9 / 3$ \\
1 & 8 & $182 /(5 \times 7)$ \\
2 & 20 & $861 /(5 \times 19)$ \\
3 & 56 & $109854 /(125 \times 55)$ \\
4 & 164 & $2895129 /(625 \times 163)$ \\
5 & 488 & $77327622 /(3125 \times 487)$ \\
6 & 1460 & $415448109 /(3125 \times 1459)$ \\
\hline \hline
\end{tabular}

Evolution scaling for trapping time. We begin by recording the numerical values of $T_{i}^{g}$. Obviously, for all $g \geq 0, T_{1}^{g}=0$; for $g=0$, it is a trivial case, we have $T_{2}^{0}=T_{3}^{0}=T_{4}^{0}=3$. For $g \geq 1$, the values of $T_{i}^{g}$ can be obtained straightforwardly via equation (77). Table 2 lists the numerical values of $T_{i}^{g}$ for some nodes up to $g=6$. The numerical values listed in table 2 show that for a given node $i$ we have $T_{i}^{g+1}=\frac{9}{5} T_{i}^{g}$. That is to say, upon growth of the Apollonian network from $g$ to generation $g+1$, the mean time to first reach the trap increases by a factor $\frac{9}{5}$. This is a basic character of random walks on the Apollonian network, which can be established from the arguments below $[1,25]$.

Consider an arbitrary node $i$ in the Apollonian network $\mathbb{A}_{g}$ after $g$ generation evolution. From equation (2), we know that upon growth of the Apollonian network to generation $g+1$, the degree $k_{i}$ of node $i$ doubles. Thus, at generation $g+1$, node $i$ has $k_{i}$ old neighbors (born at generation $g$ or earlier) and $k_{i}$ new neighbors (generated at generation $g+1$ ). Let the mean transmit time for going from node $i$ to any of its $k_{i}$ old neighbors be $X$; and let the mean transmit time for going from any of its $k_{i}$ new neighbors to one of the $k_{i}$ old neighbors be $Y$. Then we can establish the following underlying backward equations

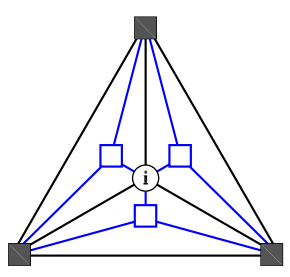

Fig. 5: Growth of trapping time in going from $\mathbb{A}_{g}$ to $\mathbb{A}_{g+1}$. Node $i \in \mathbb{A}_{g}$ has $k_{i}$ neighbor nodes in generation $g(\boldsymbol{\square})$ and $k_{i}$ new neighbor nodes in generation $g+1(\square)$. A new node has a degree of 3 , and is linked to $i$ and its two old neighbor nodes.

(see figure 5)

$$
X=\frac{1}{2}+\frac{1}{2}(1+Y), \quad Y=\frac{2}{3}+\frac{1}{3}(1+X),
$$

which leads to $X=\frac{9}{5}$. That is to say, the passage time from any node $i\left(i \in \mathbb{A}_{g}\right)$ to any node $j\left(j \in \mathbb{A}_{g}\right)$ increases by a factor of $\frac{9}{5}$, upon the network growth from generation $g$ to generation $g+1$. Thus, we have $T_{i}^{g+1}=\frac{9}{5} T_{i}^{g}$, which will be useful for deriving the formula for the mean firstpassage time in the following text.

Formula for the mean first-passage time. Having obtained the scaling of mean transmit time for old nodes, we now determine the mean first-passage time, with an aim to derive an exact solution. We represent the set of nodes in $\mathbb{A}_{g}$ as $\Omega_{g}$, and denote the set of nodes created at generation $g$ by $\bar{\Omega}_{g}$. Thus we have $\Omega_{g}=\bar{\Omega}_{g} \cup \Omega_{g-1}$. For the convenience of computation, we define the following quantities for $m \leq g$ :

$$
T_{m, \text { total }}^{g}=\sum_{i \in \Omega_{m}} T_{i}^{g},
$$

and

$$
\bar{T}_{m, \text { total }}^{g}=\sum_{i \in \bar{\Omega}_{m}} T_{i}^{g} .
$$

Then, we have

$$
T_{g, \text { total }}^{g}=T_{g-1, \text { total }}^{g}+\bar{T}_{g, \text { total }}^{g} .
$$

Next we will explicitly determine the quantity $T_{g \text {,total }}^{g}$. To this end, we should firstly determine $\bar{T}_{g \text {,total }}^{g}$. 
We examine the mean time to absorption for the first several generations of Apollonian network. In the case of $g=1$, by the very construction of Apollonian network, it follows that

$$
\begin{aligned}
& T_{5}^{1}=\left(1+T_{1}^{1}\right) / 3+\left(1+T_{2}^{1}\right) / 3+\left(1+T_{3}^{1}\right) / 3, \\
& T_{6}^{1}=\left(1+T_{1}^{1}\right) / 3+\left(1+T_{3}^{1}\right) / 3+\left(1+T_{4}^{1}\right) / 3, \\
& T_{7}^{1}=\left(1+T_{1}^{1}\right) / 3+\left(1+T_{2}^{1}\right) / 3+\left(1+T_{4}^{1}\right) / 3, \\
& T_{8}^{1}=\left(1+T_{2}^{1}\right) / 3+\left(1+T_{3}^{1}\right) / 3+\left(1+T_{4}^{1}\right) / 3 .
\end{aligned}
$$

Thus,

$$
\begin{aligned}
\bar{T}_{1, \text { total }}^{1} & =\sum_{i \in \bar{\Omega}_{1}} T_{i}^{1}=T_{5}^{1}+T_{6}^{1}+T_{7}^{1}+T_{8}^{1} \\
& =4+\left(T_{1}^{1}+T_{2}^{1}+T_{3}^{1}\right)=4+\bar{T}_{0, \text { total }}^{1} .
\end{aligned}
$$

Similarly, for $g=2$ case, it is easy to obtain

$$
\begin{aligned}
\bar{T}_{2, \text { total }}^{2} & =\sum_{i \in \bar{\Omega}_{2}} T_{i}^{2}=\sum_{i=9}^{20} T_{i}^{2} \\
& =4 \times 3+2 \bar{T}_{0, \text { total }}^{2}+\bar{T}_{1, \text { total }}^{2} .
\end{aligned}
$$

Proceeding analogously, it is not difficult to derive that

$$
\begin{aligned}
\bar{T}_{g, \text { total }}^{g}=4 \times 3^{g-1} & +\bar{T}_{g-1, \text { total }}^{g}+2 \bar{T}_{g-2, \text { total }}^{g}+\ldots \\
& +2^{g-2} \bar{T}_{1, \text { total }}^{g}+2^{g-1} \bar{T}_{0, \text { total }}^{g}(14)
\end{aligned}
$$

and

$$
\begin{aligned}
\bar{T}_{g+1, \text { total }}^{g+1}=4 \times 3^{g} & +\bar{T}_{g, \text { total }}^{g+1}+2 \bar{T}_{g-1, \text { total }}^{g+1}+\ldots \\
& +2^{g-1} \bar{T}_{1, \text { total }}^{g+1}+2^{g} \bar{T}_{0, \text { total }}^{g+1},
\end{aligned}
$$

where $4 \times 3^{g-1}$ and $4 \times 3^{g}$ are actually the numbers of nodes generated at generations $g$ and $g+1$, respectively. Equation (15) minus equation (14) times $\frac{18}{5}$ and making use of the relation $T_{i}^{g+1}=\frac{9}{5} T_{i}^{g}$, one gets

$$
\bar{T}_{g+1, \text { total }}^{g+1}=\frac{27}{5} \bar{T}_{g, \text { total }}^{g}-\frac{4}{5} \times 3^{g} .
$$

Using $\bar{T}_{1, \text { total }}^{1}=\frac{101}{5}$, equation (16) is solved inductively

$$
\bar{T}_{g, \text { total }}^{g}=\frac{32}{9}\left(\frac{27}{5}\right)^{g}+3^{g-1} .
$$

Substituting equation (17) for $\bar{T}_{g \text {,total }}^{g}$ into equation (11) and using $T_{g-1, \text { total }}^{g}=\frac{9}{5} T_{g-1, \text { total }}^{g-1}$, we have

$$
T_{g, \text { total }}^{g}=\frac{9}{5} T_{g-1, \text { total }}^{g-1}+\frac{32}{9}\left(\frac{27}{5}\right)^{g}+3^{g-1} .
$$

Considering the initial condition $T_{1, \text { total }}^{1}=\frac{182}{5}$, equation (18) is resolved by induction to yield

$$
T_{g, \text { total }}^{g}=\frac{3^{g}}{6 \times 5^{g}}\left(17 \times 3^{g}+5^{g+1}+32 \times 9^{g}\right) .
$$

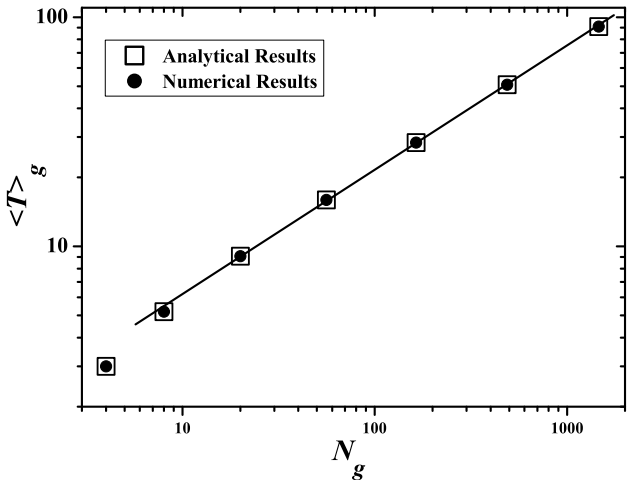

Fig. 6: Mean first-passage time $\langle T\rangle_{g}$ versus network order $N_{g}$ on a log-log scale. The solid line is a guide to the eye.

Plugging the last expression into equation (7), we arrive at the accurate formula for the average of the mean time to absorption at the trap located at node 1 on the $g$ th of Apollonian network:

$$
\langle T\rangle_{g}=\frac{3^{g}\left(17 \times 3^{g}+5^{g+1}+32 \times 9^{g}\right)}{6 \times 5^{g}\left(2 \times 3^{g}+1\right)} .
$$

We have checked our analytic formula against numerical values quoted in Table 1 For the range of $0 \leq g \leq 6$, the values obtained from equation (20) completely agree with those numerical results on the basis of the direct calculation through equation (77). This agreement serves as an independent test of our theoretical formula.

From equation (11), we have $g=\log _{3}\left(\frac{N_{g}}{2}-1\right)$. Hence, for large network (i.e., $N_{g} \rightarrow \infty$ ), we obtain

$$
\langle T\rangle_{g} \sim\left(\frac{9}{5}\right)^{g}=\left(\frac{N_{g}}{2}-1\right)^{2-\frac{\ln 5}{\ln 3}} \sim N_{g}^{(2-\ln 5 / \ln 3)},
$$

where the exponent $2-\frac{\ln 5}{\ln 3} \approx 0.535<1$. Thus, in the large limit of network order $N_{g}$, the MFPT increases algebraically with increasing order of the network.

Generally, finite-size effect plays a crucial role in critical phenomena and dynamical processes on networks [45]. In order to study the finite-size effect in the scaling behavior of MFPT for the Apollonian network, we plot a figure to show the MFPT with order for small networks, see figure 6. Surprisingly, it is observed from figure 6 that the finite-size effect has little influence on the scaling.

Previous studies showed that for regular lattices [10], Sierpinski fractals $[15,16]$, and T-graph [17] with large order $N$, their MFPT $\langle T\rangle$ behaves as $\langle T\rangle \sim N^{\alpha}$ with $\alpha>1$. Even in a complete graph of $N$ nodes, $K_{N}$, the MFPT $\langle T\rangle$ grows linearly with $N$ as $\langle T\rangle=N-1$. Actually, linear scaling of the MFPT with $N$ is the best that has been reported so far. From equation (21), one can see that the MFPT $\langle T\rangle$ of the Apollonian network increases as a fractional power of network order $N$, which implies that Apollonian network has a faster transmit time than any other analytically soluble media. In other words, the Apollonian network has the best structure for fast diffusion one can see heretofore. 
We argue that the heterogeneous topology of the Apollonian network may be responsible for the high efficiency for transport. In the Apollonian network, there are some nodes with high degree, which are linked to one another and to most other nodes in the network. These 'large' nodes can be easily visited by a walker starting from an arbitrary location. Since 'large' nodes, including the trap node, are connected to one another, so the walker can find the trap in a very short time.

Conclusions. - In summary, we have studied trapping problem on the Apollonian network exhibiting remarkable features (scale-free behavior and small-world effects) of a variety of real networks. We have obtained an analytical closed-form solution for the MFPT for random walks with a trap located at a hub node, which is consistent with the numerical computation. The rigorous result indicates that the MFPT on the Apollonian network shows a very distinct behavior, which is compared with those results previously reported for regular lattices, Sierpinski fractals, T-graph, and complete graph. We have shown that Apollonian network is the most efficient network for transport by diffusion, in contrast to other studied structure. We hope that the current study may cast some light on trapping problem in some real networks, that present similar topologies as the Apollonian network.

Acknowledgment. - This research was supported by the National Basic Research Program of China under grant No. 2007CB310806, the National Natural Science Foundation of China under Grant Nos. 60704044, 60873040 and 60873070, Shanghai Leading Academic Discipline Project No. B114, and the Program for New Century Excellent Talents in University of China (NCET-060376).

\section{REFERENCES}

[1] S. Havlin and D. ben-Avraham, Adv. Phys. 36, 695 (1987).

[2] R. Metzler and J. Klafter, J. Phys. A: Math. Gen. 37, R161 (2004).

[3] R Burioni and D Cassi, J. Phys. A: Math. Gen. 38, R45 (2005).

[4] I. M. Sokolov, J. Mai, and A. Blumen, Phys. Rev. Lett. 79, 857 (1997).

[5] J. Noolandi, Phys. Rev. B 16, 4466 (1977).

[6] A. L. Lloyd, and R. M. May, Science, 292, 1316 (2001).

[7] F. Fouss, A. Pirotte, J. M. Renders, and M. Saerens, IEEE Trans. Knowl. Data Eng. 19, 355 (2007).

[8] S. Condamin, O. Bénichou, V. Tejedor, R. Voituriez, and J. Klafter, Nature (London) 450, 77 (2007).

[9] S. Condamin, V. Tejedor, R. Voituriez, O. Bénichou and J. Klafter, Proc. Natl. Acad. Sci. USA 105, 5675 (2008).

[10] E. W. Montroll, J. Math. Phys. 10, 753 (1969).

[11] S. B. Yuste and K. Lindenberg, Chem. Phys. 284, 169 (2002).

[12] C. Loverdo, O. Bénichou, M. Moreau, and R. Voituriez, Nature Phys. 4, 134 (2008).
[13] O. Bénichou, M. Coppey, M. Moreau, P.-H. Suet, and R. Voituriez, Phys. Rev. Lett. 94, 198101 (2005).

[14] M. F. Shlesinger, Nature (London) 443, 281 (2006).

[15] J. J. Kozak and V. Balakrishnan, Phys. Rev. E 65, 021105 (2002).

[16] J. J. Kozak and V. Balakrishnan, Int. J. Bifurcation Chaos Appl. Sci. Eng. 12, 2379 (2002).

[17] E. Agliari, Phys. Rev. E 77, 011128 (2008).

[18] R. Albert and A.-L. Barabási, Rev. Mod. Phys. 74, 47 (2002).

[19] A.-L. Barabási and R. Albert, Science 286, 509 (1999).

[20] D. J. Watts and H. Strogatz, Nature (London) 393, 440 (1998).

[21] M. E. J. Newman, SIAM Rev. 45, 167 (2003).

[22] S. N. Dorogovtsev, A. V. Goltsev and J.F.F. Mendes, Rev. Mod. Phys. 80, 1276 (2008).

[23] J. D. Noh and H. Rieger, Phys. Rev. Lett. 92, 118701 (2004).

[24] V. Sood, S. Redner, and D. ben-Avraham, J. Phys. A: Math. Gen. 38, 109 (2005).

[25] E. M. Bollt, D. ben-Avraham, New J. Phys. 7, 26 (2005).

[26] A. Baronchelli, M. Catanzaro, and R. Pastor-Satorras, Phys. Rev. E 78, 011114 (2008).

[27] A. G. Cantú and E. Abad, Phys. Rev. E 77, 031121 (2008).

[28] A. Kitts, S. Carmi, S. Havlin, and P. Argyrakis, EPL 84, 40008 (2008).

[29] J.S. Andrade Jr., H.J. Herrmann, R.F.S. Andrade and L.R. da Silva, Phys. Rev. Lett. 94, 018702 (2005).

[30] J.P.K. Doye and C.P. Massen. Phys. Rev. E 71, 016128 (2005).

[31] S. S. Manna and H.J. Herrmann, J. Phys. A: Math. Gen. 24, L481 (1991).

[32] Z.Z. Zhang, F. Comellas, G. Fertin and L.L. Rong, J. Phys. A: Math. Gen. 39, 1811 (2006).

[33] Z. Z. Zhang, L. L. Rong, and S. G. Zhou, Phys. Rev. E, 74, 046105 (2006).

[34] Z. Z. Zhang, L. C. Chen, S. G. Zhou, L. J. Fang, J. H. Guan, and T. Zou, Phys. Rev. E 77, 017102 (2008).

[35] T. Zhou, G. Yan, and B.H. Wang, Phys. Rev. E 71, 046141 (2005).

[36] Z.-G. Huang, X.-J. Xu, Z.-X. Wu, and Y.-H. Wang, Eur. Phys. J. B 51, 549 (2006).

[37] Y. Hayashi and J. Matsukubo, Phys. Rev. E 73, 066113 (2006).

[38] A. A. Moreira, D. R. Paula, R. N. C. Filho, and J. S. Andrade, Jr., Phys. Rev. E 73, 065101(R) (2006).

[39] P. G. Lind, L. R. da Silva, J. S. Andrade jr., and H. J. Herrmann, EPL 78, 68005 (2006).

[40] V. Schwämmle, M. C. González, A. A. Moreira, J. S. Andrade, Jr., and H. J. Herrmann, Phys. Rev. E 75, 066108 (2007).

[41] A. P. Vieira, J. S. Andrade, Jr., H. J. Herrmann, and R. F. S. Andrade, Phys. Rev. E 76, 026111 (2007).

[42] I. N. de Oliveira, F. A. B. F. de Moura, M. L. Lyra, J. S. Andrade, Jr., and E. L. Albuquerque, Phys. Rev. E 79, 016104 (2009).

[43] C. N. Kaplan, M. Hinczewski, and A. N. Berker arXiv: 0811.3437.

[44] J. G. Kemeny and J. L. Snell, Finite Markov Chains (Springer, New York, 1976).

[45] H. Hong, M. Ha,. and H. Park, Phys. Rev. Lett. 98, 
Random walks on Apollonian network with a single trap

258701 (2007). 